\title{
Should positive sentinel node cases have a further axillary dissection?
}

\author{
B. Cady \\ Department of Surgery, Rhode Island Hospital, RI, USA.
}

\begin{abstract}
The treatment of lymph node metastasis in human cancers has been a source of controversy for many years. Several contemporary randomized trials indicate no survival advantage to performing axillary dissection in contrast to merely observing the axilla after invasive breast cancer. This assumption is confirmed since survival advantages cannot be demonstrated when comparing greater or lesser lymph node dissections or dissection vs. observation of regional lymph nodes in melanoma and breast cancer and all other epithelial cancers.

Many recent studies indicate that axillary metastases, when they do occur in this era of mammographic screening and small invasive cancers, demonstrate metastases in the axillary sentinel nodes only in from $20 \%$ to almost $100 \%$ depending on primary cancer size. For instance, in T1a and T1b cancers, $50-100 \%$ of lymph node metastases, infrequently discovered, are contained entirely within the sentinel nodes. In T2 cancers, this proportion is roughly $25-50 \%$, and in T3 cancers it ranges from $20 \%$ to $40 \%$. A number of recent articles have demonstrated no difference in survival and very little nodal recurrence when patients with positive sentinel nodes are not followed by axillary dissection although the axillary disease tends to be less advanced in observed compared to treated patients with axillary dissection. Nevertheless, basic principles have been reconfirmed in these reports.

It has also been shown in animal research that clones of particular metastatic site cells demonstrate organ specificity. Transplanted human breast cancers demonstrate specific homing abilities to selective distant metastatic organ sites such as lymph nodes, bone, lung, or even adrenal gland. Genetic profiles of the different clones of cells with high metastatic specificity are each unique. Thus it is assumed that lymph node specific metastatic cells (and other organ-site-specific cells) have no ability to lodge and grow in other metastatic sites; this has been demonstrated clinically in clinical trials previously mentioned.

Therefore, there is no benefit in performing subsequent axillary dissections even when the sentinel node biopsy is positive, because of: (1) very low risk $(\leqslant 2 \%)$ of recurrent clinical nodal metastases, (2) the lack of impact on disease specific or overall survival, (3) most lymph node metastases are limited to the sentinel nodes, and (4) lymph node metastases are only indicators not governors of the distant metastases. The American College of Surgeons Oncology Group (ACOSOG) Z-11 Trial was designed to specifically address this point, but unfortunately was closed early because of poor accrual; nevertheless, 900 patients were enrolled and will be analyzed over time to address this point.
\end{abstract}

Keywords: Axillary dissection; Lymph node metastases; Sentinel node biopsy

Correspondence to: B. Cady, MD, Department of Surgery, Rhode Island Hospital, 593 Eddy Street, APC 4, Providence, RI 02903, USA. E-mail: bcady@usasurg.org; Tel: 401 4446158; Fax: 4014446681

Work attributed to: Department of Surgery, Rhode Island Hospital, 593 Eddy Street, APC 4, Providence, RI 02903, USA

Received: 16/11/05

Accepted: 18/05/06

First published online 29/06/06

BCO/504/2005/FO 


\section{Should positive sentinel node cases have a further axillary dissection?}

The question raised by the title is a controversial topic in management of breast cancer at the present time. It is important to understand the historical background of nodal disease in breast cancer. In the 1930s and 1940s, two-thirds of patients had axillary nodal metastases, usually multiple [1]. By the 1960s and 1970s, that proportion was down to roughly $50 \%$, but still largely involved multiple nodal metastases [1]. Beginning in the late 1980s with the onset of extensive mammographic screening, the proportion of patients with positive axillary nodes has declined still further. In the entire state of Rhode Island in the years 19872003 , encompassing the wide spread use of sentinel node biopsy, the rate of metastatic nodes in all breast cancer patients was only 26\% [2]. Among cancers that were discovered just by mammography, that positive node rate is in the order of $15 \%$ or less [3]. Currently $44 \%$ of node positive patients in Rhode Island have only one-node metastases, and only a minority have more than three-node metastases [2]. Many of the positive nodes in these mammographically discovered cancers, and in smaller palpable cancers, are micrometastasis ( $2 \mathrm{~mm}$ or less in diameter). The implication of such small nodal micrometastases is still somewhat unclear, but in node metastases less than $0.2 \mathrm{~mm}$ in diameter, usually detected by immunohistochemical (IHC) staining, there is apparently no prognostic implication. Thus the American Joint Commission on Cancer (AJCC) in the most recent 6th edition has defined such minor collections of cancer cells in lymph nodes as $\mathrm{N}_{0}\left(\mathrm{HC}^{+}\right)$and urged that such a node definition not be used in prognostication or decision-making in breast cancer [4].

Thus over the past 50 years there have been enormous changes in the burden of lymph node metastases such that node metastases now occur in the minority of patients, and that in a majority of contemporary patients that do have nodal metastases, there is only minimal involvement. On this background, of course, the introduction of sentinel node biopsy has been possible, particularly as a method of avoiding axillary dissection entirely in patients that have negative sentinel nodes. Sentinel node biopsy thus has had a dramatic effect on the management of breast cancer by essentially eliminating the need for any further axillary investigation or axillary dissection in the great majority of patients who have no nodal metastases. In fact, when sentinel nodes are positive, in over $60 \%$ of patients, these are the only axillary nodes involved, indicating that sentinel node removal itself is sufficient to remove all metastases in the majority of situations [5]. That proportion of patients who have nodal metastases confined only to the sentinel nodes varies by size of the primary cancer: in $\mathrm{T}_{2}$ cases from $25 \%$ to $50 \%$, and in $\mathrm{T}_{3}$ cases from $20 \%$ to $40 \%$.

When the sentinel node is positive, a variety of factors need to be appreciated to make decisions regarding subsequent axillary dissection. First, it is critical that pathologists exactly define the 'positive' axillary node. Is it a collection of cells $\leqslant 0.2 \mathrm{~mm}$ in maximum size detected by $\mathrm{IHC}$, in which the staging is $\mathrm{N}_{0}\left(\mathrm{HC}_{\mathrm{HC}}+\right)$ ? Is the metastasis larger than $.2 \mathrm{~mm}$ but $\leqslant 2 \mathrm{~mm}$, usually detected by routine hematoxylin and eosin (H\&E) staining, in which case it is $N_{1 \text { (mic) }}$ with prognostic implications that are uncertain? While metastases larger than $2 \mathrm{~mm}$ in diameter may be meaningful prognostically, the issue is whether there are other node metastases in the axilla and whether they need removal. This incidence of other metastases is related to the size of the primary cancer, the number of sentinel nodes and non-sentinel nodes removed and the number and size of our nodal metastases discovered, as well as other factors such as age and grade [6]. Thus if a patient has a single positive macrometastases but four negative other sentinel or non-sentinel nodes, the likelihood of finding other node metastases in the axilla is extremely unlikely. However, if the patient had a single sentinel node discovered and it was positive with a macrometastases, then the possibility of other node metastases in the axilla may range up to $50 \%$ depending on primary tumor characteristics such as size, grade, lymph vessel invasion, receptor activity, etc. A nomogram has been developed and validated to calculate the risk of other metastases in non-sentinel nodes [6].

Recent literature has indicated that even if a sentinel node is positive and a follow-up axillary dissection is not performed, the risk of a regional axillary nodal recurrence following breast conservation and radiation therapy is only about $2 \%$ [7]. Interestingly, this nodal recurrence rate is similar to regional nodal recurrence after axillary dissection. Even if the axilla is not specifically treated by regional lymphatic radiotherapy, there is a similar very low rate of nodal recurrence. When breast conservation is practiced with accompanying radiotherapy, the tangent radiation portals for the breast cover the lower $1 / 2$ to $2 / 3$ of the axillary lymph nodes in anatomic studies performed [8]. Thus the majority of lower axillary lymph nodes, whether positive or negative, are actually treated in the usual breast conservation patient with whole breast radiation therapy as a result.

What regional nodal therapy to undertake after defining a positive sentinel node should be contingent on the expected survival of the patient, the expected regional recurrence rate, and the implication of other possible nodal metastases. Would any major alteration 
of management depend on knowledge about the remaining axillary lymph nodes? Would radiation portals be adjusted or added? Would different chemotherapeutic agents be utilized?

To understand lymph node metastases, the purpose of the lymphatic system should be known [9]. The functioning lymphatic system is the principal implementing organ of immunological competence of the host. From an evolutionary point of view lymphocytes and their collection in lymph nodes were a later development of the method of more carefully interrogating contamination from the hostile external environment by viruses, parasites, and bacteria. Immunocompetence develops with humoral antibodies from B lymphocytes (innate immunological system), or the rapidly changing interactions with the hostile external environment that produces cellular responses from $\mathrm{T}$ lymphocytes with cytotoxicity from cytokines and other products (adoptive immune system) [10]. The lymphatic system has only two other functions: the return of interstitial fluid to the vascular space and the delivery of nutrients from the intestinal tract to the vascular space, both via the lymphatic vessels that eventually coalesce and form the thoracic duct. Thus lymphocytes and lymph nodes are entirely related to the need for an immunological defense from the external environment and, developmentally, have almost nothing to do with tumors since cancers are, with some exceptions, almost entirely 'self' rather than 'other'. Few human cancers elicit significant antibody responses either naturally or when manipulated in the laboratory (melanoma, neuroblastoma, renal cell carcinoma and others) and the overwhelming majority of human cancers do not normally elicit significant immunological responses. Thus lymph node metastases are an accidental by-product of cancer cells shed into the lymphatic stream without an immunological reaction.

The other aspect of evaluating whether one should remove possible lymph node metastases derives from analysis of extensive clinical and laboratory literature regarding the relationship of lymph node metastases to survival $[9,11]$. In summary, there is no consistent evidence that removing lymph node metastases in a wide variety of cancers (gastric, esophageal, lung, colon, rectum, breast, head and neck, and other sites) improves survival, although it definitely decreases regional nodal recurrence rates. In randomized trials in breast, gastric, lung, esophagus, and colon cancer, survival results are similar whether the regional node basins are observed, sampled, dissected, or treated with radiation therapy [11]. Greater or lesser nodal resections in pancreatic, gastric, colon, esophageal, or lung cancer show no difference in survival [11]. Observation vs. routine initial regional node dissection in patients with melanoma [12] and breast cancer [13] show no improvement in survival overall. The concern that deliberately leaving behind lymph node metastases might impair survival in breast cancer has been addressed by at least six recent randomized clinical trials [14]. When patients are randomized between observation or dissection of axillary lymph nodes, there is no difference in long-term survival, either overall or disease-free, even though a high proportion $(30-50 \%)$ of the breast cancer patients observed are known to have node metastases by the incidence of node metastases in the arm randomized to nodal resection. In these six randomized trials comparing axillary dissection to observation in breast cancer patients, disease-free and overall survival is identical. These studies demonstrate that lymph node metastases are 'indicators but not governors' of distant metastases and survival, and this assumption is continually borne out in the clinical literature.

While lymph node metastases are an excellent prognostic indicator, in terms of indicating the statistical likelihood of distant metastases, they themselves do not govern outcomes, since lymphatic metastases of whatever extent do not interfere with any vital organ function such as metastases to the liver, brain, or lung. Survival is governed entirely by such distant metastases to vital organs, not by metastases to non-vital organs. Cells from lymph node metastases may only have the capacity to grow in nodal sites, and animal research indicates that human breast cancer node metastatic cells when injected into animals go only to lymph node sites [15], an exquisite example of metastatic specificity [16-19]. Many research studies clearly demonstrate this metastatic specificity phenomenon. Thus lymph node metastases are not generating foci for vital organ metastases.

Other recent research that bears on the question of whether to dissect the axilla after a positive sentinel node is derived from the research laboratories, clinical studies, and the organ transplantation literature. Utilizing organs for transplantation from donors who have ever had an invasive cancer transmits a significant risk of transmitting that donor cancer to the recipient. These transplanted cancers clearly must arise from occult or dormant cells in the donated organ after recipient immunosuppression. Thus, donors that are disease-free after melanoma treatment transmitting that melanoma to recipients after kidney transplantation is well documented in the literature, even many years after the melanoma was removed [20]. Such transplanted melanoma and the resulting clinical metastases cause death in half the recipients. Such transplanted melanoma may not kill the recipient if immunosuppression is withdrawn, which results in rejection of the transplanted kidney, but usually also the transplanted melanoma. Lung 
and/or heart organ donations transplant cancers from donors who have ever had an invasive cancer to almost $50 \%$ of recipients [21]. Such invasive malignancies transmitted with donor organs have arisen from a variety of organ sites including cervix, kidney, liver, lung, prostate cancer, brain tumors, and sarcomas. This phenomenon is important in that it indicates that dissemination of individual cancer cells or cancer cell clusters, even from small cancers and cured cancers, is extremely common but usually unaccompanied by a worsened outcome because of the dormant nature of such transmitted cancer cells. Thus in T1a and T1b breast cancers, bone marrow aspiration reveals cancer cells in 25\% and 35\% of patients [22], although 15-year disease-free survival is $95 \%$ and $90 \%$, respectively. The frequency of these metastatic cells in the bone marrow replicates the cells that would have been seen in transplanted donor organs in recipients of transplantation if they had been sampled in as detailed a fashion as lymph nodes or bone marrow. A recent [23] report from M.D. Anderson Hospital documents that 7-22 years after mastectomy for breast cancer, there are circulating cancer cells in over $1 / 3$ of patients that are clinically completely disease-free, indicating the frequency of 'dormant' metastases that reside for long periods of time without clinical expression if angiogenesis and progressive growth does not occur. All this recent data puts the implication of cancer cells seen in the lymph node in perspective. Since we do not pathologically or histologically sample other organs as minutely as we do sentinel nodes, we do not detect such cells, clusters of cells, or micrometastases in other solid organs. Thus many minor or limited nodal metastases may not be progressive, do not serve as generating foci for other organ specific metastatic cells, do not destroy any vital organ function themselves, and serve only as a marker for metastatic potential.

In summary, there is no need to do an axillary dissection following the discovery of a positive sentinel node unless the information gleaned from such an axillary dissection would change, in a definitive way, the therapy proposed. If biopsy of a single sentinel node revealed a macrometastases and subsequent axillary dissection revealed no further metastases, extensive nodal radiation could be avoided, that might be a reason to do the axillary dissection. If the subsequent axillary dissection revealed four positive nodes, an appropriate judgement would include comprehensive nodal radiation therapy, since such patients have a high risk of regional nodal recurrence and poor survival.

Thus the answer to the original question is complex and multifaceted depending on patient and cancer features and what therapeutic measures might result from the additional information obtained by the axillary dissection. Increasingly, however, the need for axillary dissection, even after a positive sentinel node biopsy, should be seriously questioned, and will be abandoned entirely over the next few years [24].

\section{References}

1. Cady B, Stone M, Schuler J, et al. The new era in breast cancer. Arch Surg 1996; 131: 301-308.

2. Coburn N, Chung MA, Fulton J, Cady B. Decreased breast cancer tumor size, stage, and mortality in Rhode Island: an example of a well-screened population. Cancer Control 2004; 11(4): 222-230.

3. Hunt K, Rosen E, Sickles E. Outcome analysis for women undergoing annual versus biennial screening mammography: a review of 24,211 examinations. Am J Roentgenol 1999; 173(2): 285-289.

4. Breast. In: Greene FL, Page DL, Fleming ID, et al. (Eds). AJCC Cancer Staging Handbook. Greene, FL SpringerVerlag; New York 2002.

5. Viale G, Maiorano E, Pruneri G, et al. Predicting the risk for additional axillary metastases in patients with breast carcinoma and positive sentinel lymph node biopsy. Ann Surg 2005; 241(2): 319-325.

6. Lambert L, Ayers G, Hwang R, et al. Validation of a breast cancer nomogram for predicting nonsentinel lymph node metastases after a positive sentinel node biopsy. Ann Surg Oncol 2006; 13(3): 310-320.

7. Naik A, Fey J, Gemignani M, et al. The risk of axillary relapse after sentinel lymph node biopsy for breast cancer is comparable with that of axillary lymph node dissection. Ann Surg 2004; 240(3): 462-471.

8. Chung MA, DiPetrillo T, Hernandez S, et al. Treatment of the axilla by tangential breast radiotherapy in women with invasive breast cancer. Am J Surg 2002; 184: 401-402.

9. Cady B. Is axillary lymph node dissection necessary in routine management of breast cancer? NO. Principle Pract Oncol 1998; 12(7): 1-12.

10. Basic immunobiology: a primer. In: Berke G, Clark W (Eds). Killer Lymphocytes. New York: Springer; 2005: 1-28.

11. Gervasnoi J, Taneja C, Chung MA, Cady B. Biologic and clinical significance of lymphadenectomy. Surg Clin N Am 2000; 80. 1631-1673

12. Balch CM, Soong S-J, Bartolucci A, et al. Efficacy of an elective regional lymph node dissection of 1 to $4 \mathrm{~mm}$ thick melanomas for patients 60 years of age and younger. Ann Surg 1996; 224(3): 263-266.

13. Fisher B, Jeong J, Anderson S, et al. Twenty-five year follow-up of a randomized trial comparing radical mastectomy, total mastectomy, and total mastectomy followed by irradiation. New Engl J Med 2002; 347: 567-575.

14. Cady B. A randomized trial comparing axillary dissection to no axillary dissection in elderly patients with T1N0 breast cancer. Ann Surg 2005; 242(1): 7-9.

15. Nip J, Shibata H, Loskutoff D, et al. Human melanoma cells derived from lymphatic metastases use integrim AvB3 to adhere to lymph node vitronectin. J Clin Invest 1992; 90: 1406.

16. Woelfle U, Cloos J, Sauter G, et al. Molecular signature associated with bone marrow micrometastasis in human breast cancer. Cancer Res 2003; 63: 5679-5684. 
17. Espana L, Fernandez Y, Rubio N, et al. Overexpression of $\mathrm{Bcl}-\mathrm{XI}$ in human breast cancer cells enhances organselective lymph node metastasis. Breast Cancer Res Treat 2004; 87: 33-44.

18. Skobe $M$, Hawighorst $T$, Jackson $D$, et al. Induction of tumor lymphangiogenesis by VEGF-C promotes breast cancer metastasis. Nat Med 2001; 7(2): 192-198.

19. Karpanen $T$, Egeblad M, Karkkainen $M$, et al. Vascular endothelial growth factor $\mathrm{C}$ promotes tumor lymphangiogenesis and intralymphatic tumor growth. Cancer Res 2001; 61:1786-1790.

20. MacKie R, Reid R, Junor B. Fatal melanoma transferred in a donated kidney 16 years after melanoma surgery. New Engl J Med 2003; 348(6): 567-568.
21. Buell J, Trofe J, Hanaway M, et al. Transmission of donor cancer into cardiothoracic transplant recipients. Surgery 2001; 130(4): 660-666 discussion 666-668.

22. Braun S, Vogl F, Naume B, et al. A pooled analysis of bone marrow micrometastasis in breast cancer. New Engl J Med 2005; 353: 793-802.

23. Meng S, Tripathy D, Frenkel EP, et al. Circulating tumor cells in patients with breast cancer dormancy. Clin Cancer Res 2004; 10: 8152-8162.

24. Cady B. The process of clinical metastases in cancer: contemporary animal research and clinical reports suggest unifying concepts. Submitted to Ann Surg Oncol 2006. 